\title{
The measurement of the reactor antineutrino flux and spectrum of Daya Bay Experiment
}

\section{Fengpeng An* (for the Daya Bay Collaboration)}

East China University of Science and Technology

E-mail: anfengpeng@gmail.com

The Daya Bay Reactor Neutrino Experiment collected 300,000 inverse beta decay events in three antineutrino detectors at two sites near the reactor cores, over 217 days. This paper will present the methods we use to convert the observed positron energy spectrum to a reactor antineutrino spectrum, with an universal unit $\mathrm{cm}^{2} /$ fission $/ \mathrm{MeV}$. We also present our results for the absolute reactor antineutrino flux and spectrum. Comparisons are made with the predictions of various flux models, an example of using our spectrum to predict the spectrum from other reactor experiments will also be described.

16th International Workshop on Neutrino Factories and Future Neutrino Beam Facilities - NUFACT2014, 25 -30 August, 2014

University of Glasgow, United Kingdom

\footnotetext{
* Speaker.
} 


\section{Introduction}

The Daya Bay Experiment is designed to precisely measure the neutrino mixing angle $\sin ^{2} 2 \theta_{13}$ with a near-far strategy [1,2]. The experiment deploy 8 identical antineutrino detectors (AD) in the Daya Bay NPP, which consists of 6 reactor cores each with $2.9 \mathrm{GW}$ thermal power. The layout of the experiment is shown in Fig. 1. The ADs are installed in 3 experimental halls (EH), of which $\mathrm{EH} 1$ and $\mathrm{EH} 2$ are the near sites and EH3 is the far site.

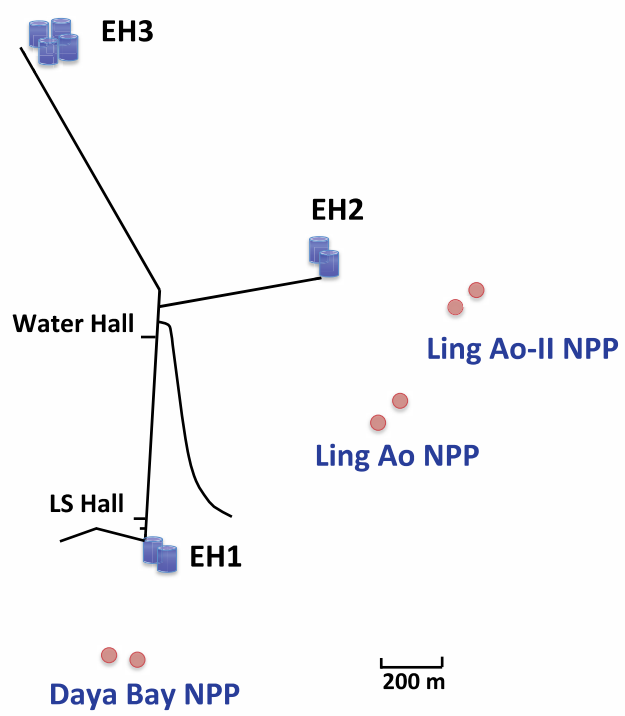

Figure 1: Layout of the Daya Bay experiment. The red dots represent reactors, the blue cylinders represent the antineutrino detectors installed in three EHs.

The antineutrinos are detected via inverse beta decay (IBD) interactions in the ADs. The experiment used a 6-AD data taking strategy from 24 December 2011 to 28 July 2012, with 2 ADs in EH1, $1 \mathrm{AD}$ in EH2 and 3 ADs in EH3. The experiment began the full operation on October 19, 2012, and in the full operation each near site has 2 ADs and the the far site has 4 ADs.

During the 6-AD period of 217 days, more than 300,000 IBD events were collected by the near site detectors, which made the largest sample of antineutrinos events among short-baseline reactor antineutrino experiments. The data of the 6-AD period were analyzed to give direct measurement of absolute reactor antineutrino flux and spectrum and compared with predictions with reactor isotope models. A generic reactor antineutrino spectrum was deduced from the absolute measurement to be used for the prediction of future reactor antineutrino experiments.

\section{The measurement of absolute reactor antineutrino flux and spectrum}

The Daya Bay experiment recently reported the analysis result of $\bar{v}_{e}$ rate and detected energy spectrum during the 6-AD data taking period [3]. This analysis was based on the comparison of the relative rates or spectrum of $\bar{v}_{e}$ between the near sites and the far site. Compared to this previous analysis, in current study the absolute detection efficiency has been improved to give the absolute measurement of the rate and spectrum. The improvements include: the Gd capture ratio, 
delayed energy cut, spill-in correction, and their uncertainties. The improved detection efficiency is determined to be $80.59 \% \pm 2.08 \%$.

A slightly different rate-only fitter from [1] is constructed to obtain the absolute flux:

$$
\chi^{2}=\sum_{d=1}^{6} \frac{\left[M_{d}-T_{d}\left(1+\varepsilon_{R}+\varepsilon_{D}+\sum_{r} \omega_{r}^{d} \alpha_{r}+\varepsilon_{d}\right)+\eta_{d}\right]^{2}}{M_{d}+B_{d}}+\sum_{r} \frac{\alpha_{r}^{2}}{\sigma_{r}^{2}}+\sum_{d=1}^{6}\left(\frac{\varepsilon_{d}^{2}}{\sigma_{d}^{2}}+\frac{\eta_{d}^{2}}{\sigma_{B}^{2}}\right)+\frac{\varepsilon_{D}^{2}}{\sigma_{D}^{2}}
$$

where $M_{d}$ is the measured IBD events of the $d$ th detector. $B_{d}$ is the corresponding backgrounds of each detector, $T_{d}$ is the predicted IBD events considering the reactor flux, neutrino oscillation and the detector response by MC simulation, $\omega_{r}^{d}$ is the IBD contribution fraction of the $r$ th reactor to the $d$ th detector. $\sigma_{r}(0.8 \%)$ is the uncorrelated reactor uncertainty, $\sigma_{d}(0.2 \%)$ is the uncorrelated detection uncertainty and $\sigma_{B}$ is the background uncertainty. $\alpha_{r}, \varepsilon_{d}$ and $\eta_{d}$ are the corresponding nuisance parameters. $\varepsilon_{R}$ is the nuisance parameter for the reactor absolute flux normalization, which is floating. $\varepsilon_{D}$ is for the absolute uncertainty of the detection efficiency, with a penalty term added into the fitter. The two free parameter are $\varepsilon_{R}$ and $\sin ^{2} 2 \theta_{13}$. The prediction is calculated with two models: ILL+Vogel [4] [5]model and Huber+Mueller model [6] [7]. Using the the ILL+Vogel (Huber+Mueller) model in the prediction the best fit results are: $\sin ^{2} 2 \theta_{13}=0.0905 \pm$ $0.0095(0.0906 \pm 0.0095)$ and $\varepsilon_{R}=-0.007 \pm 0.023(-0.053 \pm 0.022)$. The flux ratio of measurement over prediction is $\left(1-\varepsilon_{R}\right)$, the result is $0.992 \pm 0.023(0.947 \pm 0.022)$ using ILL+Vogel ( Huber+Mueller ) model.

Another method is applied to calculate the absolute flux without fitting. In this method the measured IBD events in each detector are normalized to $Y_{0}$ with unit: $\mathrm{cm}^{2}$ proton $^{-1} \mathrm{GW}^{-1}$ day $^{-1}$ and $\sigma_{f}$ with unit: $\mathrm{cm}^{2}$ fission ${ }^{-1}$. The normalization is corrected with the reactor power and fission fractions of all reactors, and the oscillation effect with the best-fit $\sin ^{2} 2 \theta_{13}$. The result of the near-site combined absolute flux is $Y_{0}=1.533 \times 10^{-18} \mathrm{~cm}^{2}$ proton ${ }^{-1} \mathrm{GW}^{-1}$ day $^{-1}$ and $\sigma_{f}=5.934 \times$ $10^{-18} \mathrm{~cm}^{2}$ fission $^{-1}$.

The effective baseline is $573 \mathrm{~m}$, which is calculated flux weighted detector-reactor distances of the 3 ADs in near sites. The effective fission fractions are calculated flux weighted fission fractions to $3 \mathrm{ADs}$ at two near halls, defined as the total antineutrino number contribution of a certain fissile isotope $i$ over all the reactor antineutrinos:

$$
f_{i}=\frac{F_{i}}{F_{\text {total }}}
$$

where $F_{i}$ is the baseline weighted total fission number of fissile isotope $i$ from all reactors "observed" by the $3 \mathrm{ADs}$, and $F_{\text {total }}$ is the baselined weighted total fission number of all isotopes from all reactors "observed" by the 3 ADs. Both were calculated with daily thermal powers, isotope fission fractions and baselines. The result of the effective fissions:

${ }^{235} U:{ }^{238} U:{ }^{239} \mathrm{Pu}:{ }^{241} \mathrm{Pu}=0.586: 0.076: 0.288: 0.050$.

The result of the absolute reactor antineutrino flux is consistent with previous short-baseline reactor experiments. Fig 2 shows the global ratio of the measurement over prediction results, which are all normalized to the Huber+Mueller flux model. The global ratio (excluding Daya Bay) R is $0.943 \pm 0.008$ and the Daya Bay measurement $\mathrm{R}$ is $0.947 \pm 0.022$.

The measured positron spectra of IBD events in the three near site ADs are combined and compared with the prediction of the same combination. The predicted antineutrino spectrum was 


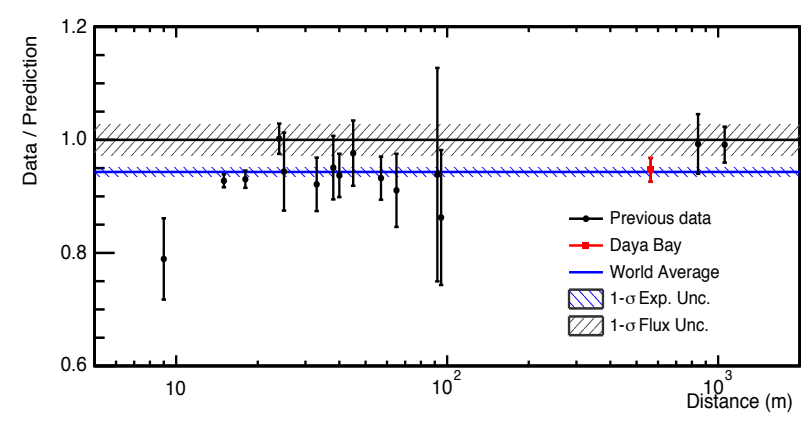

Figure 2: The measured antineutrino flux from experiments with various baselines, normalized to the Huber+Mueller flux model. The horizontal bar represents the global average and its $1 \sigma$ error band. The 3\% reactor flux uncertainty is shown as a band around unity.

transformed into prompt positron spectrum by utilizing the detector response matrix. The response matrix was built and cross checked with multiple methods. Both of the ILL+Vogel model and Huber + Muller Model were used in the prediction for comparison. The predicted spectrum was normalized to the measurement for shape only comparison. A $\chi^{2}$ function was construct to qualify the discrepancy:

$$
\begin{aligned}
& \chi^{2}=\left(N_{i}^{\text {obs }}-N_{i}^{\text {pred }}\right) V_{i j}^{-1}\left(N_{j}^{\text {obs }}-N_{j}^{\text {pred }}\right) \\
& V=V_{\text {stat }}+V_{\text {reactor }}+V_{\text {detector }}+V_{\text {bkgs }}
\end{aligned}
$$

where $N_{i}^{o b s}$ is the events in the $i$ th bin of measured prompt spectrum, and $N_{i}^{\text {red }}$ is the events in the $i$ th bin of predicted prompt spectrum, $V$ is the full covariance matrix which includes the covariance matrices of uncertainties from statistics, the reactor, the detectors and backgrounds, of which the diagonal elements are shown in Fig 3.

Fig 4 shows the comparison of the measured and predicted IBD spectra. In the top panel, the black dot is the measured IBD prompt spectrum with statistical error bars, the red dots are the predicted spectrum (Huber+Mueller model) which is normalized to the measurement with the full $1 \sigma$ error band(diagonal term of full covariance matrix, excluding statistical error). The bottom panel shows the ratio data/prediction, the gray band is the reactor uncertainty component, the red band has the same meaning with that on the top panel. The blue curve is the ratio of ILL+Vogel model predicted spectrum over the Huber+Mueller one. From the plot a bump can be clearly observed in the energy range 4-6 MeV, which is also seen by Double Chooz Experiment [9] and Reno Experiment [10]. The blue curve has a flat shape which means the bump like shape also exists between the measurement and ILL+Vogel model.

The $\chi^{2}$ comparison in Equation (2.3) gives the result $\chi^{2} / n d f=41.4 / 24$, corresponding to a $2.4 \sigma$ discrepancy.

Furthermore, another two methods were developed to quantify the significance of localized deviations. One method calculated the $\chi^{2}$ contribution from each bin:

$$
\overline{\chi_{i}}=\frac{N_{i}^{\text {obs }}-N_{i}^{\text {pred }}}{\left|N_{j}^{\text {obs }}-N_{j}^{\text {pred }}\right|} \sqrt{\frac{1}{2} \sum_{j} \chi_{i j}^{2}+\chi_{j i}^{2}}
$$




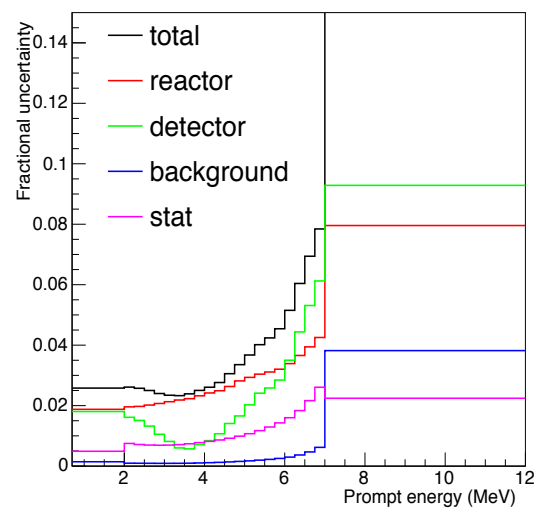

Figure 3: Diagonal elements of the full covariance matrix and its components.

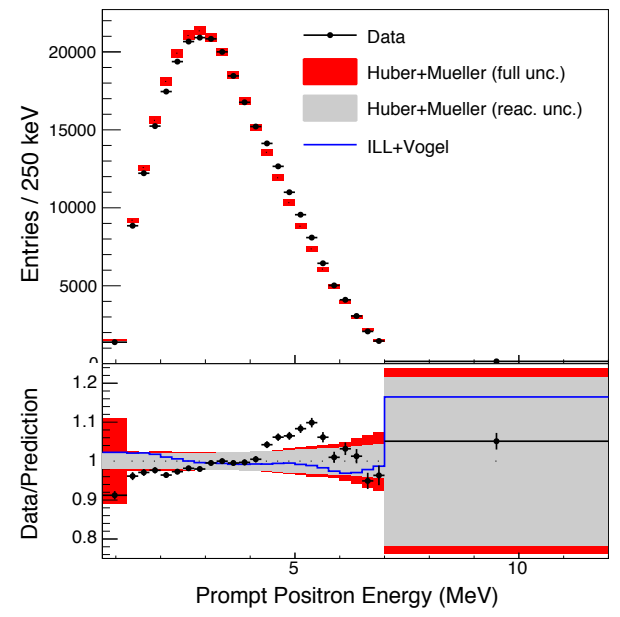

Figure 4: Comparison of near site combined measured and predicted IBD prompt spectrum with uncertainty band. Top panel: the absolute spectra comparison (Huber+Mueller model). Bottom panel: Ratio of measurement and prediction (Huber+Mueller model). Blue curve: the ratio of predictions with the ILL+Vogel model over Huber+Mueller model.

where $\chi_{i j}^{2}=\left(N_{i}^{o b s}-N_{i}^{p r e d}\right) V_{i j}^{-1}\left(N_{j}^{o b s}-N_{j}^{p r e d}\right)$. The result is plotted in panel (B) in Fig 5. The other method introduces $\mathrm{N}$ (number of bins) nuisance parameters with no pull terms to the oscillation fitter. The $\chi_{\min }^{2}$ difference before and after introducing the $\mathrm{N}$ nuisance parameters is expected to follow a $\chi^{2}$ distribution with N-1 d.o.f., therefore a P-value can be calculated. The $\Delta \chi^{2} / n d f$ with a $1 \mathrm{MeV}$ energy window is shown in the Panel (C) in Fig 5. In the energy range of 4-6 MeV (i.e., a $2 \mathrm{MeV}$ window), the P-value from the $\Delta \chi^{2} / n d f$ method is $4.66 \times 10^{-5}$, or about $4.1 \sigma$. All the comparisons implies that the discrepancy between prediction and the measurement around the energy range 4-6 $\mathrm{MeV}$ exists.

We also did some investigations of the events in 4-6 MeV, finding that:

The events are reactor power correlated and time independent as other IBD events.

The events match all IBD event characteristics: e.g., neutron capture time and distance distributions, prompt event position distribution, etc, and disfavors unexpected backgrounds.

All evidences seem to point to the prediction model, which has been pointed out by D. A. Dwyer and T. J. Langford with a abinitio calculation in a recent paper[11]: a specific set of fission daughters may explain the extra amount of neutrinos.

\section{Deduced antineutrino spectrum from Daya Bay Experiment}

An antineutrino spectrum was deduced from the Daya Bay measured spectrum. This spectrum is independent with the Daya Bay detector response and could be used as a generic spectrum to predict the reactor antineutrino flux and spectrum for other experiments. 


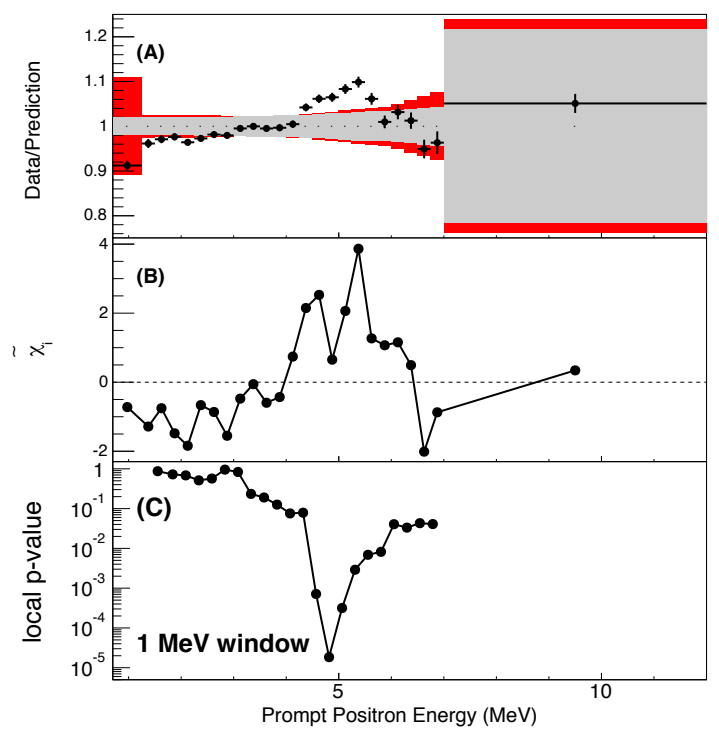

Figure 5: Panel (A): ratio of measurement and prediction of prompt energy spectrum. Panel (B): $\chi^{2}$ contribution from each bin. Panel (C): Local P-value scan with $1 \mathrm{MeV}$ energy window.

The deduction was done in two steps. Firstly, the measured IBD spectrum in the near site detectors were combined together, the combined spectrum was then converted into antineutrino spectrum by utilizing unfolding. The input of the unfolding includes: the measured IBD spectrum, the covariance matrix of measured IBD spectrum, and the detector response matrix derived from full MC. The output of the unfolding is unfolded antineutrino spectrum and the covariance matrix of the unfolded antineutrino spectrum. Multiple cross checks were done to the unfolding. Two unfolding algorithms, the Singular Value Decomposition(SVD) and Bayes iteration algorithms were applied and got almost same results. In addition, the independence of unfolded spectrum to MC input of response matrix, the minimization of variance plus bias were all tested. Secondly, the oscillation was removed from this unfolded spectrum, and then the spectrum was normalized into an universal unit: $\mathrm{cm}^{2} /$ fission/MeV:

$$
S_{\text {obs }}\left(E_{\bar{v}_{e}}\right)=\frac{S_{\text {unfolded }}\left(E_{\bar{v}_{e}}\right)}{P_{e f f}\left(E_{\bar{v}_{e}}, L\right) \cdot N_{p} \cdot F_{\text {total }}}
$$

where $S_{o b s}\left(E_{\bar{v}_{e}}\right)$ is the deduces generic antineutrino spectrum, $P_{e f f}$ is the flux and baseline weighted survival probability of the neutrinos from six cores, $N_{p}$ is the total proton numbers in the detectors, $F_{\text {total }}$ is the calculated total fission numbers in all reactors. The deduced generic spectrum is plotted in Fig 6. As a comparison, the predicted spectrum of the near site antineutrino spectrum was processed with the same steps as in Equation (3.1) and was plotted in Fig 6. The bottom panel of Fig 6 is the ratio of the measured and predicted spectrum. It has the same rate deficit as the flux measurement and similar spectral deviations as in the comparison of measured and predicted IBD prompt spectra. The deduced antineutrino spectrum of Daya Bay also supplies data outside $[2,8]$ $\mathrm{MeV}$, and it naturally contains the "extra" portion of antineutrinos in 4-6 MeV which the predicted spectrum doesn't have. The uncertainty of the spectrum is under further estimation. 


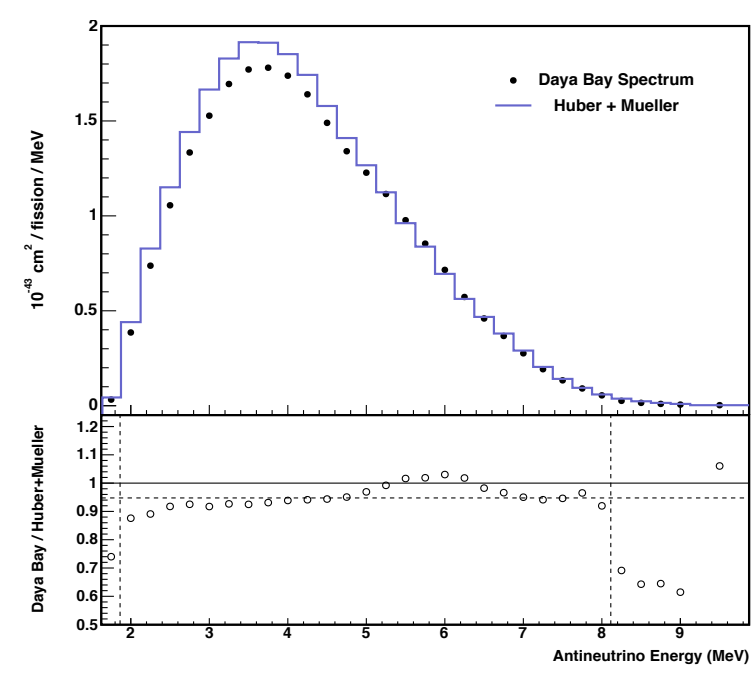

Figure 6: Top panel: Data points: the deduced reactor antineutrino spectrum from the measurement of Daya Bay. Blue curve: the predicted spectrum with the Huber+Mueller model. Bottom panel: ratio of the measurement and the prediction. The horizontal dashed line is at 0.947 , when normalizing the measurement to the Huber+Mueller model. the prediction with the Huber+Mueller model outside the vertical dashed lines is from extrapolation.

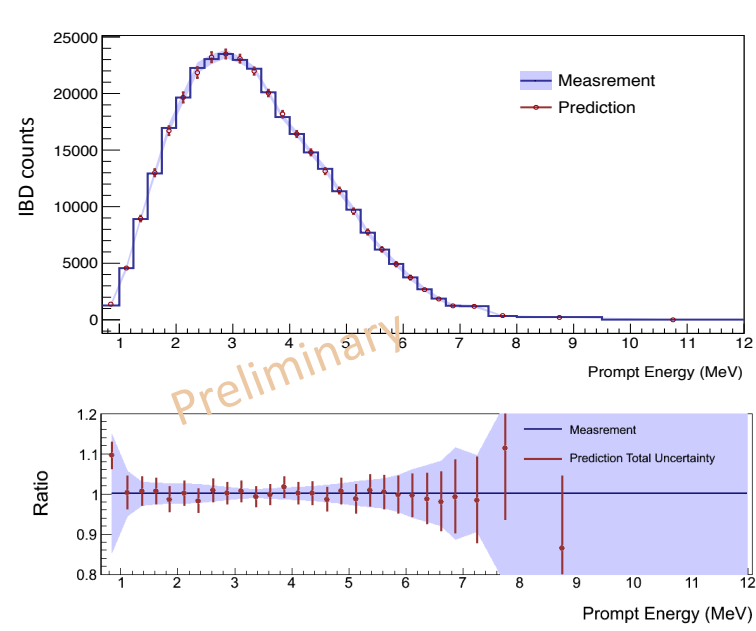

Figure 7: Top panel: Blue line: the measured prompt IBD spectrum of "Experiment $X$ " with full uncertainty, red line: the predicted spectrum by utilizing Daya Bay deduced generic spectrum. Bottom panel: the ratio of the prediction over the measurement, with the full uncertainty bar(band). The effective fission fraction of "Experiment $X$ " is ${ }^{235} U:{ }^{238} U::^{239}$ $P u:{ }^{241} P u=0.5292: 0.0765: 0.3303: 0.0618$

A method was developed to use the Daya Bay deduced spectrum to do prediction for other reactor experiments. Suppose there is another experiment called $X$, the reactor antineutrino spectrum of $X$ could be predicted as:

$$
S_{X}\left(E_{\bar{v}_{e}}\right)=\frac{1}{4 \pi L_{X}^{2}} \cdot F_{X} \cdot\left[S_{D Y B}\left(E_{\bar{v}_{e}}\right)+\sum_{i}\left(f_{D Y B}^{i}-\alpha_{X}^{i}\right) S_{I L L}^{i}\left(E_{\bar{v}_{e}}\right)\right]
$$

where the index $i$ indicates the fissile isotope, $L_{X}$ is the baseline of experiment $X, F_{X}$ is the total fission number of $X, S_{D Y B}\left(E_{\bar{v}_{e}}\right)$ is the Daya Bay deduced generic spectrum, $f_{D Y B}^{i}$ is the effective fission fraction of Daya Bay, $\alpha_{X}^{i}$ is the fission fraction of $X$, and $S_{I L L}^{i}\left(E_{\bar{v}_{e}}\right)$ is the ILL model based prediction. In this formula, the shape of the spectrum is predicted by the terms in the square brackets. The most part of the spectrum shape is predicted by Daya Bay deduced spectrum $S_{D Y B}\left(E_{\bar{v}_{e}}\right)$. Because the fission fractions are different between two experiments, there is a residual part which is predicted by ILL based models, which is shown as the second term in the square brackets in formula (3.2).

A comparison was done to test the new prediction method. Firstly an independent dataset outside the 6-AD data taking period was selected, and treated as another "Experiment $X$ ", then we use the method proposed above to predict the antineutrino spectrum for the "Experiment $X$ ", lastly the measured data of this $X$ dataset was analyzed to get the measured spectrum and was compared with the prediction. The result is shown in the Fig 7. The top panel shows the spectrum 
comparison of the measured spectrum and the prediction with total uncertainty, the bottom panel is the prediction over measurement ratio with total uncertainty. As can be seen, the central values are with in $1 \sigma$ uncertainty range, and the ratio shape is flat, which means the prediction method works.

\section{Summary}

Daya Bay has made a precise flux and spectrum measurement of IBD events in three ADs of near site during the 6-AD data taking time. The absolute flux is consistent with previous measurements, The measurement over prediction ratio $R=0.947 \pm 0.022(0.993 \pm 0.023)$, using Huber+Muller (ILL + Vogel ) model as the prediction method. The absolute positron spectrum measurement is not consistent $(\sim 2.4 \sigma)$ with predictions of different reactor antineutrino models, in the range 4-6 MeV the deviation is $\sim 4.1 \sigma$. Investigation shows that the IBD events characteristics in the energy range are like other normal events. A Daya Bay deduced generic spectrum was extracted, which could serve as a new option for reactor antineutrino spectrum prediction.

\section{Acknowledgement}

Part of the work in this article is supported by the National Natural Science Foundation of China (11405057, 11390383), and by China Postdoctoral Science Foundation (2014M341). The author would like to acknowledge the Daya Bay collaborators for useful comments, especially Dr. Weili Zhong, Qingwang Zhao and Jie Zhao who helped a lot in the article.

\section{References}

[1] F. P. An et al. (Daya Bay collaboration), Phys. Rev. Lett. 108, 171803 (2012).

[2] F. P. An et al. (Daya Bay collaboration), Chinese Phys. C 37, 011001 (2013).

[3] F. P. An et al. (Daya Bay Collaboration), Phys. Rev. Lett. 112, 061801(2014).

[4] K. Schreckenbach et al. Phys. Lett. B. 160, 325 (1985), F. von Feilitzsch, A. A. Hahn and K. Schreckenbach, Phys. Lett. B. 118, 162 (1982), A. A. Hahn et al. Phys. Lett. B 218, 365 (1989).

[5] P. Vogel, G. K. Schenter, F. M. Mann, and R. E. Schenter, Phys. Rev. C 24, 1543 (1981).

[6] P. Huber, Phys. Rev. C 84, 024617 (2011), 85, 029901(E) (2012).

[7] T. Mueller et al. Phys. Rev. C 83, 054615 (2011).

[8] C. Zhang, X. Qian, and P. Vogel, Phys. Rev. D 87, 073018 (2013).

[9] Abe, J. C. dos Anjos, set al. (Double Chooz Collaboration), arXiv: 1406.7763v2.

[10] Reno Collaboration, talk at Neutrino2014.

[11] D. A. Dwyer and T. J. Langford : arXiv:1407.1281 